\title{
Predicting Adverse Outcomes in Monochorionic-Diamniotic Twins: The Role of Intertwin Discrepancy in Middle Cerebral Artery Doppler Measurements and the Cerebroplacental Ratio
}

\author{
${ }^{1}$ Division of Maternal Fetal Medicine, Department of Obstetrics and \\ Gynecology, David Geffen School of Medicine at University of \\ California Los Angeles, Los Angeles, California \\ ${ }^{2}$ Division of Maternal Fetal Medicine, Center for Fetal Medicine and \\ Women's Ultrasound, Los Angeles, California \\ ${ }^{3}$ Department of Medicine Statistics Core, David Geffen School of \\ Medicine, Los Angeles, California
}

Thalia Mok, MD ${ }^{1}$ Yalda Afshar, MD, PhD ${ }^{1} \quad$ Lawrence D. Platt, MD ${ }^{1,2}$ Rong Guo, MS 3

Rashmi R. Rao, MD ${ }^{1}$ Ilina D. Pluym, MD ${ }^{1}$ Neil S. Silverman, MD ${ }^{1,2}$ Christina S. Han, MD ${ }^{1,2}$

\begin{abstract}
Address for correspondence Thalia Mok, MD, Division of Maternal Fetal Medicine, Department of Obstetrics and Gynecology, David Geffen School of Medicine at University of California Los Angeles, 10833 Le Conte Avenue, Box 951740, Los Angeles, CA 90095-1740 (e-mail: tmok@mednet.ucla.edu).
\end{abstract}

Am J Perinatol 2021;38:1348-1357.

\author{
Abstract \\ Keywords \\ - intertwin discrepancy \\ - monochorionic- \\ diamniotic twins \\ - middle cerebral \\ artery \\ - cerebroplacental \\ ratio \\ - Doppler
}

Objective This study was aimed to evaluate the role of intertwin discrepancy in middle cerebral artery peak systolic velocity (MCA-PSV) and cerebroplacental ratio (CPR) for the prediction of adverse outcomes in monochorionic-diamniotic (MCDA) twin pregnancies. Study Design A retrospective cohort study of MCDA pregnancies that underwent ultrasound surveillance at a perinatal referral center from 2007 to 2017. Intertwin MCAPSV discrepancy (MCA- $\Delta_{\text {PSV-MoM }}$ ) was defined as the absolute difference of MCA-PSV multiple of the median (MoM) for gestational age between twins. Intertwin CPR discrepancy (CPR- $\Delta$ ) was defined as the absolute difference of CPR between twins. The maximum MCA$\Delta_{\text {PSV-Mom }}$ and CPR- $\Delta$ before and after 26 weeks of gestation were assessed as predictors of pregnancy and neonatal outcomes through simple logistic regression models and Pearson's correlation coefficients. Receiver operating characteristic (ROC) curves were generated to determine the predictive value of maximum MCA- $\Delta_{\text {PSV-MoM }}$ and CPR- $\Delta$.

Results A total of 143 MCDA pregnancies met inclusion criteria. There was a significant association between MCA- $\Delta_{\text {PSV-MoM }}$ at $<26$ weeks and the development of twin anemia-polycythemia sequence (TAPS; $p=0.007$ ), intrauterine fetal demise (IUFD; $p=0.009)$, and neonatal intensive care unit (NICU) admission $(p<0.05)$. MCA$\Delta_{\text {PSV-Mom }}$ at $\geq 26$ weeks was associated with the development of TAPS $(p<0.001)$. CPR$\Delta$ at $<26$ weeks was associated with the development of twin-twin transfusion syndrome (TTTS; $p=0.03$ ) and NICU admission $\left(p=0.02\right.$ ). MCA- $\Delta_{\text {PSV-MoM }}$ at $\geq 26$ weeks was highly predictive of TAPS (area under curve $[A U C]=0.92$ ). A cut-off of 0.44 would identify TAPS with $100 \%$ sensitivity and $73 \%$ specificity. received

November 16, 2020

accepted after revision

June 17, 2021

published online

July 19, 2021 (c) 2021. Thieme. All rights reserved.

Thieme Medical Publishers, Inc.,

333 Seventh Avenue, 18th Floor,

New York, NY 10001, USA
DOI https://doi.org/

$10.1055 / \mathrm{s}-0041-1732456$. ISSN 0735-1631. 
Conclusion In MCDA pregnancies, intertwin MCA and CPR discrepancies are associated with adverse pregnancy and neonatal outcomes, including TAPS, TTTS, IUFD, and NICU admission. Evaluation of intertwin MCA and CPR differences demonstrated the potential for clinical predictive utility in the surveillance of MCDA twin pregnancies.

\section{Key Points}

- Intertwin discrepancy of MCA-PSV and CPR is associated with adverse pregnancy outcomes.

- Intertwin differences in Doppler ultrasound may occur prior to meeting diagnostic criteria for TTTS or TAPS.

- There is potential clinical predictive utility in MCA and CPR surveillance of MCDA twin pregnancies.

Monochorionic-diamniotic (MCDA) twin pregnancies are at a three to five times higher risk of perinatal morbidity and mortality than dichorionic twins. ${ }^{1}$ This increase in risk is attributed to a shared placenta and characterized by intertwin vascular anastomoses which, when unbalanced, leads to complications specific to MCDA twins. ${ }^{2,3}$ These risks include twin-twin transfusion syndrome (TTTS), twin anemia-polycythemia sequence (TAPS), and selective intrauterine growth restriction (sIUGR). ${ }^{4}$

Due to the increased risk of complications, these pregnancies require close surveillance. However, the evidence for timing and components of monitoring is limited and the recommendations for surveillance vary between governing bodies. $^{4-7}$ The Society for Maternal-Fetal Medicine recommends serial ultrasound surveillance at every 2 weeks, starting at 16 weeks, to monitor amniotic fluid levels and bladder filling per guidance from the North American Fetal Therapy Network, but there continues to be no consensus on the inclusion of umbilical artery (UA) and middle cerebral artery (MCA) Doppler measurements in the surveillance of MCDA pregnancies. ${ }^{7}$ Doppler interrogation of the UA and MCA have been shown to play an important role in antenatal assessment of high-risk pregnancies, decreasing perinatal morbidity and mortality. ${ }^{8-11}$ In addition, the cerebroplacental ratio (CPR) may have value in the assessment of fetal wellbeing and prediction of adverse neonatal outcomes. ${ }^{12-16}$ CPR is a combined measurement of fetal response and placental status and theorized to be a more sensitive Doppler index for predicting perinatal outcomes. ${ }^{17,18}$

The data on the utility of Doppler measurements in MCDA twins and prediction of adverse pregnancy outcomes are limited with variable conclusions. Some have demonstrated an association between abnormal Doppler measurements in monochorionic twins with neonatal morbidity, preterm delivery, and low birth weight (BW), while others demonstrated that isolated Doppler abnormalities are commonly observed in monochorionic twins and are not associated with adverse outcomes. ${ }^{19,20}$ Gaziano et al was the first study to evaluate CPR in both monochorionic and dichorionic twin pregnancies and demonstrated that CPR was superior to the UA and MCA in prediction of adverse neonatal events. ${ }^{21}$ Lastly, two retrospective studies have explored the novel utility of intertwin discrepancy of CPR and MCA-PSV and demonstrated an association with perinatal loss and sIUGR, respectively. $^{22,23}$

Doppler ultrasonography, specifically intertwin differences of Doppler indices, in MCDA twin pregnancies requires further exploration to identify optimal methods of surveillance and subsequent timely interventions for these highrisk pregnancies. Here, we report our evaluation of the role of intertwin differences in MCA-PSV and CPR in the surveillance of MCDA pregnancies and association with MCDA-specific complications.

\section{Materials and Methods}

We conducted a retrospective cohort study reviewing all MCDA twin pregnancies that underwent antenatal surveillance over a 10-year period between January 1, 2007, and February 1, 2017, at a single, high-volume metropolitan perinatal referral center associated with the Department of Obstetrics and Gynecology at the David Geffen School of Medicine at the University of California Los Angeles (UCLA). This study was approved by the UCLA Institutional Review Board (IRB no.: 17-000486) and a waiver of informed consent was obtained. Women with MCDA twin pregnancies who were scheduled for biweekly ultrasounds, or as clinically indicated, between 16 and 34 weeks were included. All ultrasound studies were performed by registered diagnostic sonographers, followed by a confirmatory scan by a boardcertified maternal-fetal medicine specialist. During each biweekly ultrasound, patients underwent Doppler evaluation of UA and MCA parameters, along with amniotic fluid and growth assessments per our center's protocol. Patients were identified through a search function within the clinical ultrasound database. We performed a multilayer search using dropdown values for number of gestation ("2"), chorionicity ("monochorionic-diamniotic"), and report comments for appropriate terms ("twin," "twins," and "monochorionic"). Each chart was reviewed individually to ensure accuracy of chorionicity, and clinical information and ultrasound variables were extracted from the review of medical records by one obstetrician (T.M.) to ensure uniformity in coding. Only deidentified information was used.

Pregnancies were dated by measurement of crown-rump length (CRL) of the larger twin in the first trimester, dates of 
embryo transfer in in vitro fertilization (IVF) patients, or by the date of a certain last menstrual period (LMP) in women with regular cycles, unless there was a discrepancy of more than 5 to 7 days between dating by LMP and the CRL of the larger twin. ${ }^{6}$ Chorionicity was determined by the presence of lambda or $\mathrm{T}$ signs and number of placentas at the first ultrasound. ${ }^{24}$ Exclusion criteria included pregnancies complicated by chromosomal abnormalities or major structural anomalies and patients who presented after intrauterine fetal demise (IUFD) before 16 weeks of either twin or after the development of MCDA-twin-specific complications, including TTTS, TAPS, and sIUGR. Patients with incomplete ultrasound records or without MCA Doppler measurements were also excluded.

Variables collected included maximum vertical pockets of amniotic fluid in each sac, estimated fetal weight, UA, and MCA Doppler measurements. The head circumference, biparietal diameter, abdominal circumference, and femur length were used to calculate estimated fetal weight via Hadlock's formula. ${ }^{25}$ UA Doppler measurements were categorized as normal, elevated systolic-to-diastolic ratio defined as greater than 95th percentile for gestational age, absent-end diastolic flow, or reverse-end diastolic flow. MCA Doppler measurements were defined as elevated if peak systolic velocity (PSV) was greater than 1.5 multiples of the median (MoM) for gestational age. ${ }^{26}$

Intertwin MCA-PSV discrepancy (MCA- $\Delta_{\text {PSV-MoM}}$ ) was defined as the absolute difference of MCA-PSV MoM between the twins. CPR was calculated as the ratio of MCA PI to UA PI, and intertwin CPR discrepancy (CPR- $\Delta$ ) was defined as the absolute difference of CPR between the twins. Measurements were performed between 14 to 37 weeks. For this study, the MCA- $\Delta_{\text {PSV-MoM }}$ and CPR- $\Delta$ measurements were divided evenly into two groups defined as the $<26$ weeks group (14-25 completed weeks) and the $\geq 26$ weeks group (26-37 completed weeks) and assessed as predictors of adverse pregnancy outcomes. If multiple measurements were performed, the maximum intertwin discrepancy for MCA and CPR were used for analysis.

The primary outcome of interest for this study was the development of MCDA-specific complications, including TTTS, TAPS, and sIUGR. TTTS was diagnosed according to Quintero staging criteria. ${ }^{27}$ TAPS was defined as MCA-PSV higher than 1.5 MoM for gestational age in one twin and lower than $0.8 \mathrm{MoM}$ in the other twin. ${ }^{28}$ This definition is based on our regional fetal surgeon's diagnostic criteria for TAPS which is required before fetal interventions will be offered. sIUGR was diagnosed when the estimated fetal weight of one twin was below 10th percentile for the assigned gestational age. ${ }^{29}$ Secondary outcomes that were collected included pregnancy and neonatal outcomes. Pregnancy outcomes included IUFD(s), gestational age at delivery, and mode of delivery. Neonatal outcomes included average birth weight (BW), BW difference, and NICU admission. BW difference was calculated as (larger twin BW - smaller twin BW) / larger twin BW $\times 100 \% .^{30}$

Fisher's exact test or Chi-square test was used to examine the association between categorical variables and complica- tion groups, presented as frequency count (\%). Continuous variables were presented as median (interquartile range [IQR]) and compared using the Wilcoxon's rank-sum test. The association between maximum MCA- $\Delta_{\text {PSV-MoM }}$ or CPR- $\Delta$ at $<26$ weeks and $\geq 26$ weeks and development of MCDAspecific complications and other adverse pregnancy or neonatal outcomes were evaluated through simple logistic regression models and calculation of Pearson's correlation coefficients. Receiver operating characteristic (ROC) curves were generated to assess the predictive value of maximum MCA- $\Delta_{\text {PSV-MoM }}$ and CPR- $\Delta$ at $<26$ weeks and $\geq 26$ weeks, and Youden's index was used to identify their best cut-off values in the prediction of respective outcomes. $p$-Values of $<0.05$ were considered statistically significant. All analyses were performed using SAS v9.4 (SAS Institute Inc., Cary, NC).

\section{Results}

Two hundred and twenty MCDA twin pregnancies were identified. Seventy pregnancies were excluded due to incomplete clinical data (e.g., single, second-opinion ultrasound or lack of MCA data prior to development of a standardized protocol), one was excluded due to unclear chorionicity, three were excluded due to referral after development of TTTS, and three were excluded because of major fetal structural anomalies. As a result, 143 MCDA twin pregnancies met inclusion criteria and were included in the analysis. A total of 1,763 ultrasounds were reviewed with a median of 11 (IQR: 8-14) ultrasounds performed per patient throughout the gestation. A total of 1,187 MCA and 834 CPR measurements were collected for analysis.

Baseline maternal demographics and clinical characteristics of all MCDA twin pregnancies and those that developed TTTS, TAPS, or sIUGR are depicted in - Table 1. Maternal age, body mass index (BMI), race, parity, and the presence of maternal comorbidity did not differ significantly between pregnancies complicated by MCDA-twin-specific complications. Pregnancies complicated by sIUGR were more likely to have a history of a prior preterm delivery $(p=0.02)$ and had a higher proportion of Hispanic ethnicity $(p=0.04)$. Pregnancies conceived by assisted reproductive technology did not differ in development of TTTS, TAPS, or sIUGR when compared with those that conceived spontaneously $(p>0.10)$.

Sixteen (11.2\%) pregnancies were complicated by TTTS, 7 (4.9\%) by TAPS, and $41(28.7 \%)$ by sIUGR (-Fig. 1$)$. The median gestational age at diagnosis was 22.7 weeks (range: 15.4-30.0), 30.1 weeks (range: 17.9-35.0), and 26.3 weeks (range: 14.9-25.9) for TTTS, TAPS, and sIUGR, respectively. Of those with TTTS, four (25\%) were stage I, six (37.5\%) were stage II, four (25\%) were stage III, one (6.3\%) was stage IV, and one $(6.3 \%)$ was stage $V$. Nine $(56.3 \%)$ pregnancies complicated by TTTS underwent laser ablation, and the remaining seven pregnancies underwent expectant management due to being ineligible for intervention. Five pregnancies were greater than 26 weeks of gestation at the time of diagnosis and two were complicated by IUFD of both twins. Of the seven pregnancies complicated by TAPS, six (85.7\%) were spontaneous TAPS and one (14.3\%) developed following laser 


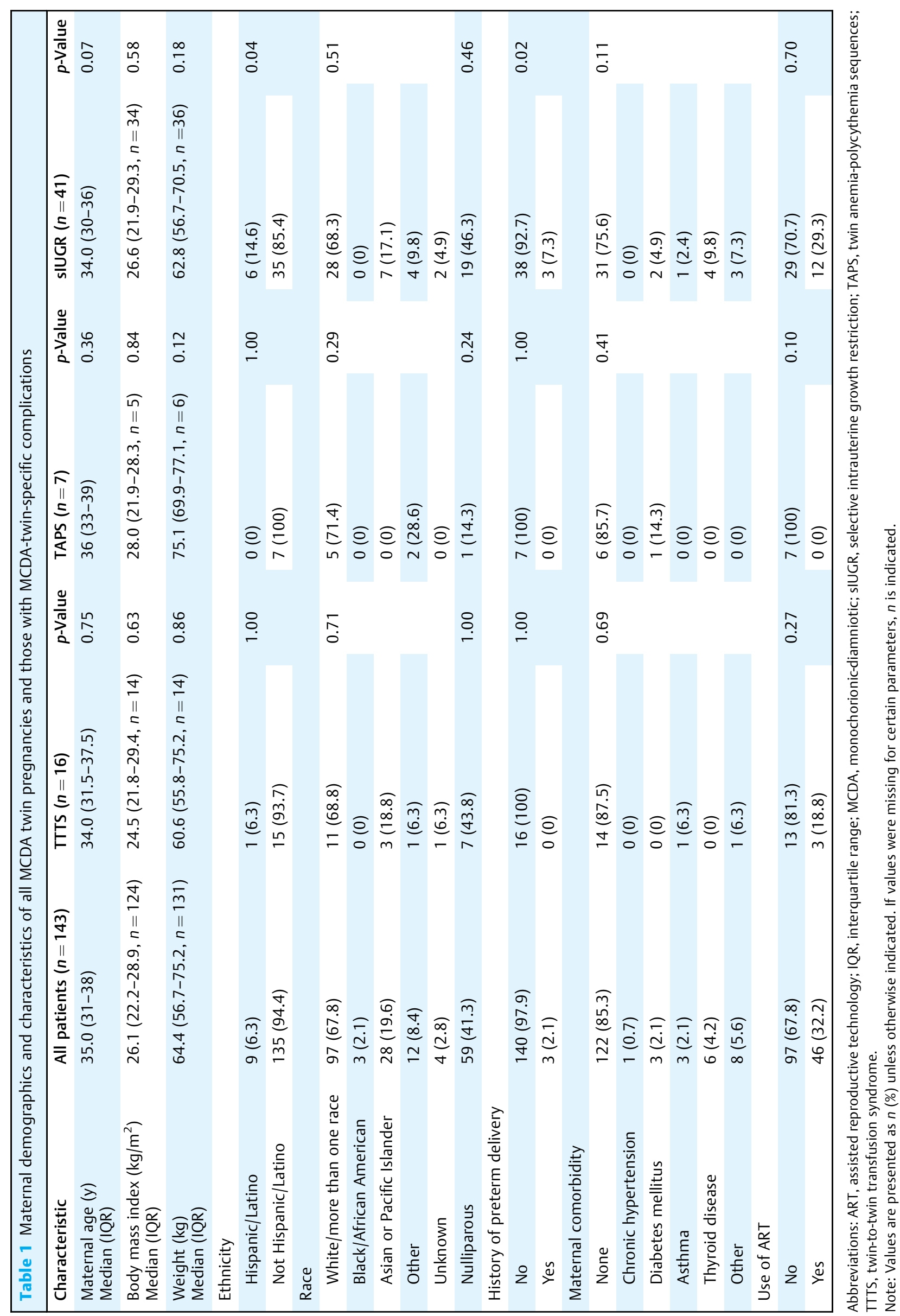


220 MCDA twin pregnancies (January 1, 2007 - February 1, 2017)

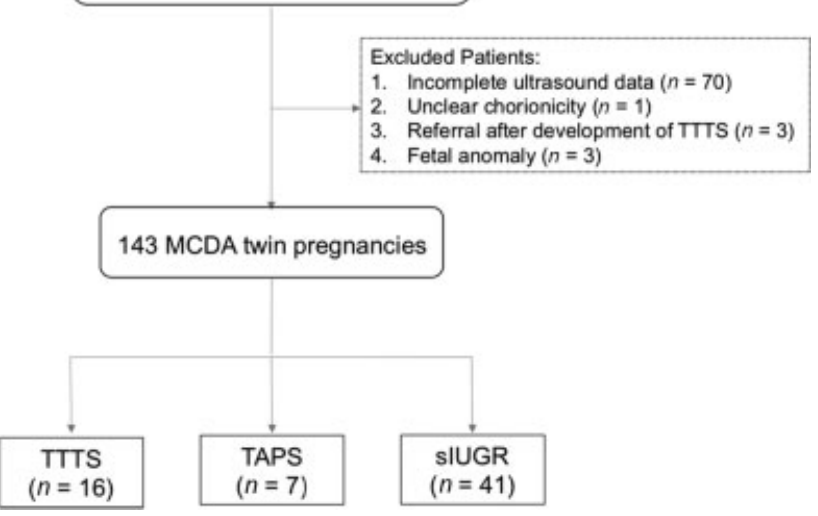

Fig. 1 Flow diagram of monochorionic-diamniotic twin pregnancies and development of primary outcomes. MCDA, monochorionic-diamniotic; sIUGR, selective intrauterine growth restriction; TAPS, twin anemia-polycythemia sequence; TTTS, twin-twin transfusion syndrome.

ablation treatment of TTTS. All but one of the pregnancies complicated by sIUGR was diagnosed with type- 1 sIUGR, and all underwent expectant management with close surveillance.

Pregnancy and neonatal outcomes are summarized in -Table 2. Fourteen patients did not have delivery outcomes available for review due to delivery outside the system, and two pregnancies were complicated by TTTS resulting in fetal demise of both twins. As a result, a total of 127 patients with pregnancy and neonatal outcomes were available for review. Gestational age of delivery was significantly lower for pregnancies complicated by TTTS $(p<0.01)$ and sIUGR $(p=0.01)$ but not for TAPS $(p=0.23)$. Indication for delivery was also significantly different for pregnancies complicated by TTTS $(p<0.01)$ and sIUGR $(p<0.01)$ but not TAPS $(p=0.46)$. There was not an increased risk of cesarean delivery for pregnancies with MCDA-specific complications. The average BW was significantly lower in pregnancies that were complicated by TTTS $(p<0.01)$ and sIUGR $(p<0.01)$ but not for pregnancies complicated by TAPS $(p=0.53)$. As expected, BW discordance was higher in pregnancies complicated by sIUGR at $17.7 \%$ (IQR: 12.1-24.0, $p<0.01$ ). Pregnancies that developed MCDA-specific complications had more than twice the NICU admissions of one or both twins compared with respective uncomplicated MCDA pregnancies. There were a total of four neonatal deaths, three of which were pregnancies complicated by TTTS.

Results of the univariate logistic regression model analysis of MCA- $\Delta_{\text {PSV-MoM }}$ and CPR- $\Delta$ are presented in - Table 3. MCA$\Delta_{\text {PSV-MoM }}$ at $<26$ weeks was associated with IUFD (odds ratio $[\mathrm{OR}]=18.91,95 \%$ confidence interval $[\mathrm{CI}]$ : 2.11-169.13, $p=0.01$,$) , NICU admission ( \mathrm{OR}=91.46,95 \% \mathrm{CI}: 1.26-$ $301.74, p=0.03$ ), and TAPS (OR=21.39, 2.33-196.80, $p=0.01)$. MCA- $\Delta_{\text {PSV-MoM }}$ at $\geq 26$ weeks was also associated with TAPS ( $\mathrm{OR}=769.79,95 \% \mathrm{CI}$ : 15.98-999.99, $p<0.01$ ). Intertwin CPR discrepancy at $<26$ weeks was significantly associated with the development of TTTS (OR $=2.23,95 \% \mathrm{CI}$ : $1.10-4.76, p=0.03)$. The average time before TTTS developed and intertwin CPR discordance first noted was 2 weeks and 1 day, and $80 \%$ of the measurements demonstrated a lower CPR value in the donor and a higher CPR value in the recipient. CPR- $\Delta$ at $<26$ weeks was also significantly associated with NICU admission of at least one twin (OR $=3.94,95 \%$ CI: $1.31-11.85, p=0.02$ ).

Pearson's correlation coefficient demonstrated a significant correlation between CPR- $\Delta$ at $<26$ weeks to gestational age at delivery $(r=-0.30, p=0.002)$ and average BW $(r=$ $-0.38, p<0.001)$. Increasing CPR- $\Delta$ at $<26$ weeks was correlated with earlier gestational age at delivery and lower average BW, respectively. There was also a moderate association of increasing CPR- $\Delta$ at $<26$ weeks with nonreassuring fetal testing requiring delivery $(r=0.39)$. In contrast, CPR- $\Delta$ at $\geq 26$ weeks was not associated with the development of complications or adverse perinatal outcomes.

ROC curves were developed to assess the predictive performance of MCA- $\Delta_{\text {PSV-MoM }}$ and CPR- $\Delta$ for MCDA-twinspecific complications and other adverse pregnancy and neonatal outcomes. -Fig. 2 presents the ROC curves for MCA- $\Delta_{\text {PSV-MoM }}$ at $<26$ weeks for TAPS (area under curve $[A U C]=0.62)$, IUFD $(A U C=0.61)$, and NICU admission $($ AUC $=0.61)$ and $\mathrm{MCA}-\Delta_{\mathrm{PSV}-\mathrm{MoM}}$ at $\geq 26$ weeks for TAPS $(A U C=0.92)$. An intertwin MCA PSV MoM discrepancy of 0.61 at $<26$ weeks would identify TAPS with a sensitivity of $57 \%$ and specificity of $98 \%$. A MCA- $\Delta_{\text {PSV-MoM }}$ cut-off of 0.27 at $<26$ weeks would identify pregnancies complicated by IUFD with sensitivity of $71 \%$ and specificity of $61 \%$, and an intertwin MCA PSV MoM discrepancy of 0.36 at $<26$ weeks would predict the requirement of NICU admission with a sensitivity of $34 \%$ and specificity of $89 \%$. With an MCA- $\Delta_{\text {PSV-MoM }}$ cut-off of 0.44 at $\geq 26$ weeks, we would identify pregnancies complicated by TAPS with a sensitivity of $100 \%$ and specificity of $73 \%$.

ROC curves for the development of TTTS (AUC $=0.68)$ and NICU admissions (AUC $=0.67$ ) by CPR $-\Delta$ at $<26$ weeks are shown in - Fig. 3. With a CPR- $\Delta$ cut-off of 0.73 at $<26$ weeks, we would identify pregnancies complicated by TTTS with a sensitivity of $67 \%$ and a specificity of $73 \%$. Using a CPR- $\Delta$ cutoff of 0.39 at $<26$ weeks, we would identify pregnancies requiring NICU admission following delivery with 78 and $55 \%$ of sensitivity and specificity, respectively.

\section{Discussion}

This study evaluates the utility of intertwin discrepancy of MCA-PSV MoM and CPR in the surveillance of MCDA twin pregnancies and its association with the development of MCDA-twin-specific complications and adverse pregnancy outcomes. We identified that increased intertwin differences in these parameters at various times in gestation can be associated with the development of complications of MCDA twin gestations and adverse pregnancy outcomes.

Confirmation that MCA- $\Delta_{\text {PSV-MoM }}$ at $\geq 26$ weeks is a strong predictor of TAPS is consistent with the current diagnostic criteria of TAPS. ${ }^{28}$ A recent study by Tollenaar et al demonstrated that delta MCA PSV $>0.5$ MoM had a greater diagnostic accuracy for predicting TAPS than the traditional 


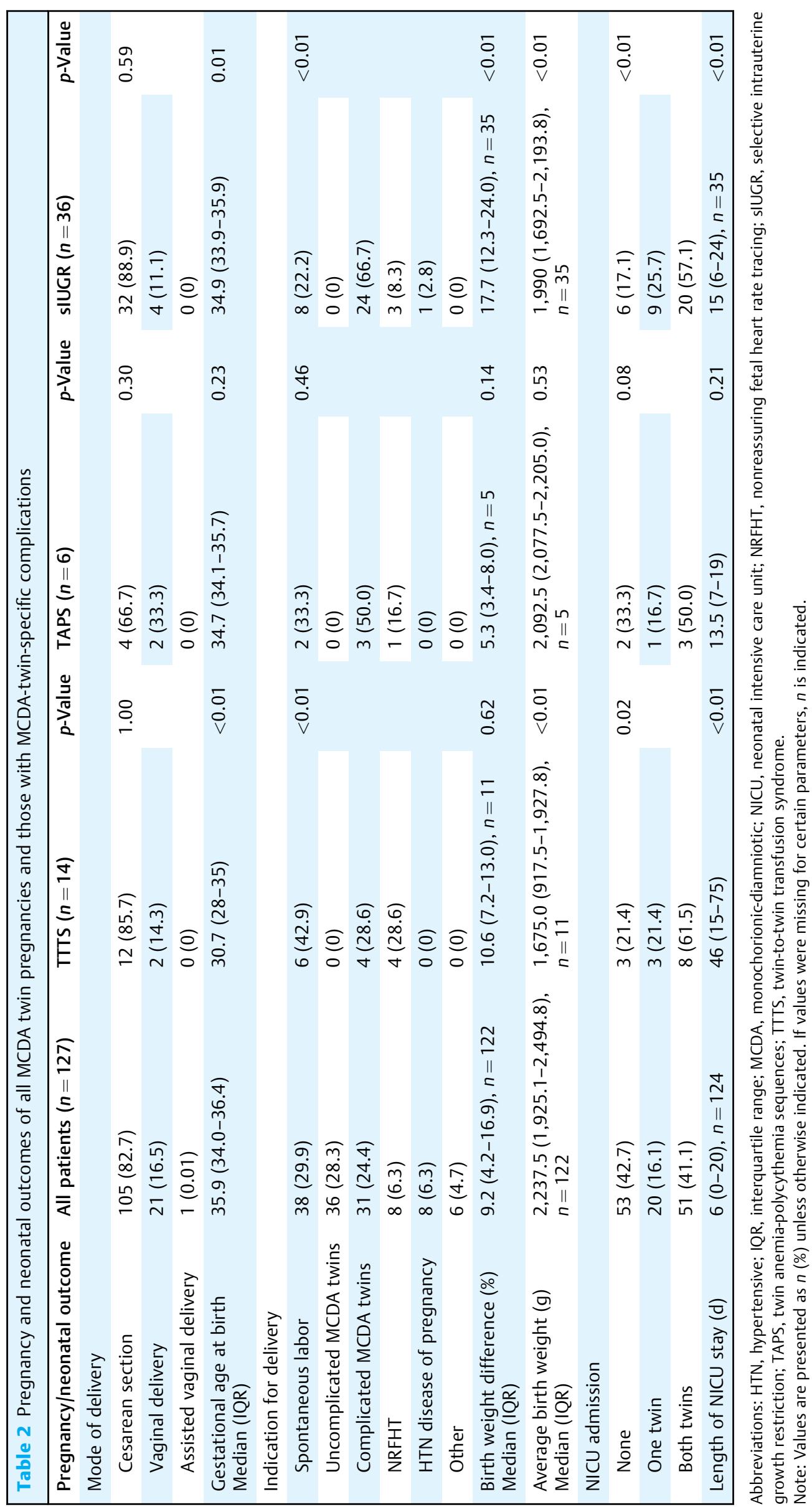




\begin{tabular}{|c|c|c|c|c|c|}
\hline Outcome & $\begin{array}{l}\text { Intertwin difference } \\
\text { with outcome }\end{array}$ & $\begin{array}{l}\text { Intertwin difference } \\
\text { without outcome }\end{array}$ & Odds ratio & $95 \% \mathrm{Cl}$ & $p$-Value \\
\hline \multicolumn{6}{|c|}{$<26$ weeks intertwin MCA-PSV MoM difference } \\
\hline TTTS & $0.35 \pm 0.32$ & $0.28 \pm 0.20$ & 3.20 & $0.48-21.33$ & 0.23 \\
\hline TAPS & $0.60 \pm 0.55$ & $0.27 \pm 0.17$ & 21.39 & $2.33-196.80$ & 0.01 \\
\hline sIUGR & $0.31 \pm 0.24$ & $0.28 \pm 0.21$ & 1.79 & $0.34-9.42$ & 0.49 \\
\hline IUFD & $0.59 \pm 0.63$ & $0.28 \pm 0.16$ & 18.91 & $2.11-169.13$ & 0.01 \\
\hline NICU admission & $0.33 \pm 0.26$ & $0.23 \pm 0.11$ & 19.46 & $1.26-301.74$ & 0.03 \\
\hline \multicolumn{6}{|c|}{$\geq 26$ weeks intertwin MCA-PSV MoM difference } \\
\hline TTTS & $0.35 \pm 0.18$ & $0.37 \pm 0.19$ & 0.88 & $0.03-24.99$ & 0.94 \\
\hline TAPS & $0.72 \pm 0.25$ & $0.35 \pm 0.17$ & 769.79 & 15.98->999.99 & $<0.01$ \\
\hline sIUGR & $0.36 \pm 0.19$ & $0.37 \pm 0.19$ & 0.78 & $0.09-6.68$ & 0.82 \\
\hline IUFD & 0.18 & $0.37 \pm 0.19$ & 0.002 & $<0.001-314.35$ & 0.31 \\
\hline NICU admission & $0.40 \pm 0.22$ & $0.33 \pm 0.15$ & 6.86 & $0.70-66.89$ & 0.10 \\
\hline \multicolumn{6}{|c|}{$<26$ weeks intertwin CPR difference } \\
\hline TTTS & $1.21 \pm 1.48$ & $0.60 \pm 0.50$ & 2.23 & $1.10-4.76$ & 0.03 \\
\hline TAPS & $0.56 \pm 0.30$ & $0.68 \pm 0.72$ & 1.09 & $0.39-3.04$ & 0.87 \\
\hline sIUGR & $0.76 \pm 0.53$ & $0.65 \pm 0.77$ & 1.23 & $0.73-2.05$ & 0.44 \\
\hline IUFD & $0.95 \pm 1.09$ & $0.64 \pm 0.67$ & 0.92 & $0.30-2.82$ & 0.80 \\
\hline NICU admission & $0.81 \pm 0.82$ & $0.50 \pm 0.40$ & 3.94 & $1.31-11.85$ & 0.02 \\
\hline \multicolumn{6}{|c|}{$\geq 26$ weeks intertwin CPR difference } \\
\hline TTTS & $0.78 \pm 0.53$ & $1.05 \pm 1.01$ & 0.58 & $0.16-2.14$ & 0.42 \\
\hline TAPS & $0.96 \pm 0.46$ & $1.03 \pm 1.01$ & 1.21 & $0.66-2.23$ & 0.55 \\
\hline SIUGR & $0.94 \pm 0.57$ & $1.06 \pm 1.09$ & 0.84 & $0.48-1.46$ & 0.53 \\
\hline IUFD & $0.58 \pm 0.37$ & $1.04 \pm 0.99$ & 0.11 & $<0.001-32.94$ & 0.44 \\
\hline NICU admission & $0.86 \pm 0.54$ & $1.20 \pm 1.44$ & 0.61 & $0.34-1.11$ & 0.11 \\
\hline
\end{tabular}

Abbreviations: Cl, confidence interval; CPR, cerebroplacental ratio; IUFD, intrauterine fetal demise; MCA-PSV, middle cerebral artery-peak systolic velocity; MCDA, monochorionic-diamniotic; MoM, multiple of the median; NICU, neonatal intensive care unit; slUGR, selective intrauterine growth restriction; TAPS, twin anemia-polycythemia sequences; TTTS, twin-to-twin transfusion syndrome.

Note: Values are presented as mean \pm standard deviation.

individual MCA PSV cut-off criteria. ${ }^{31}$ Our study supports their findings that increasing intertwin MCA- $\Delta_{\mathrm{PSV}-\mathrm{MoM}}$ may be predictive of the eventual development of TAPS. However, the MCA- $\Delta_{\text {PSV-MoM }}$ cut-off value shown to be most predictive of TAPS in our study was 0.44 MoM. This is lower than traditional diagnostic criteria for TAPS and the one suggested by Tollenaar et al. Based on our study findings, intertwin MCA- $\Delta_{\text {PSV-MoM }}$ may provide additional utility in identifying evidence of TAPS prior to meeting the classic diagnostic criteria and earlier in the process. In addition, the predictive
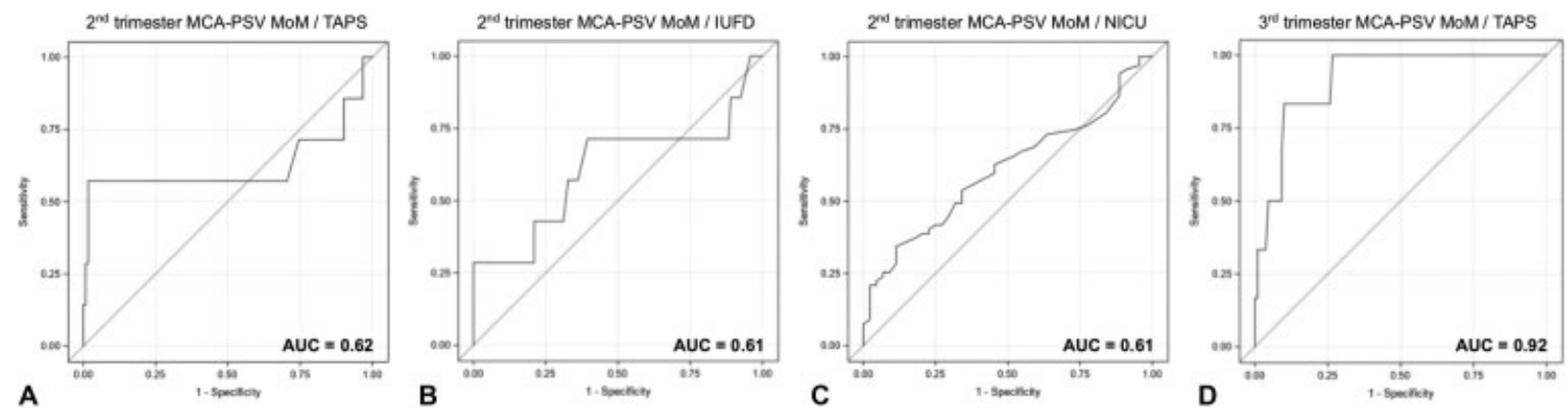

Fig. 2 Receiver operating characteristic curves for prediction of (A) TAPS, (B) IUFD, (C) NICU admission by intertwin MCA-PSV MoM difference at $<26$ weeks and (D) TAPS by intertwin MCA-PSV MoM difference at $\geq 26$ weeks. AUC, area under curve; MCA-PSV; middle cerebral artery peak systolic velocity; MoM, multiple of the median; NICU, neonatal intensive care unit; IUFD, intrauterine fetal demise; TAPS, twin anemia-polycythemia sequence. 

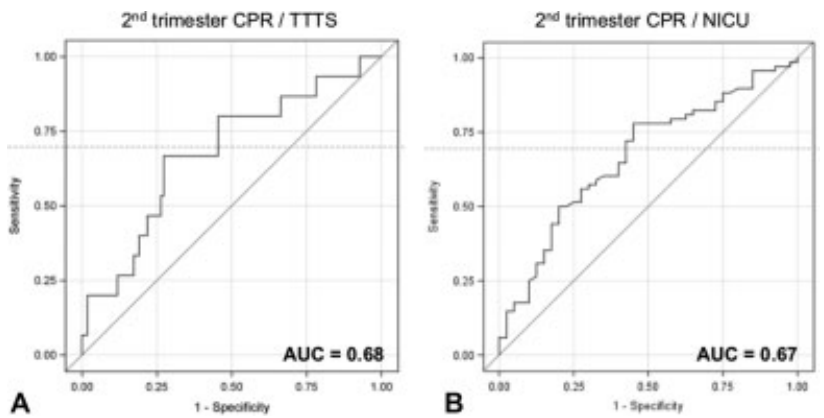

Fig. 3 Receiver operating characteristic curves for prediction of (A) TTTS and (B) NICU admission by intertwin CPR difference at $<26$ weeks. CPR, cerebroplacental ratio; NICU, neonatal intensive care unit; TTTS, twin-twin transfusion syndrome.

value of MCA- $\Delta_{\text {PSV-MoM }}$ for TAPS was significantly higher at $\geq 26$ weeks than at $<26$ weeks consistent with the majority of TAPS cases being diagnosed in the late second or third trimester. $^{32}$ The discrepancy in predictive value of MCA$\Delta_{\text {PSV-MoM }}$ at $<26$ versus $\geq 26$ weeks of TAPS argues for continued surveillance of the MCA throughout the entirety of the pregnancy.

Multiple studies have demonstrated the value of CPR in the evaluation of singleton pregnancies for the prediction of adverse perinatal outcomes, particularly in fetal growth restriction. ${ }^{13-18}$ We did not see an association with intertwin CPR difference and development of sIUGR. A possible explanation for the lack of association with sIUGR is that we did not include an estimated fetal weight discordance of $25 \%$ in the diagnostic criteria for sIUGR and as a result, the average BW discordance in our cohort was $17.7 \%$ with no pair exceeding $24 \%$ discordance, likely demonstrating less-severe sIUGR. In addition, all of the pregnancies except one were complicated by type- 1 sIUGR which is associated with a more favorable outcome. In contrast, we observed a significant association with CPR- $\Delta$ at $<26$ weeks with NICU admission. Increasing CPR- $\Delta$ at $<26$ weeks was also correlated with earlier gestational age at delivery, lower average BW, and development of nonreassuring fetal status requiring delivery. It is possible that these were confounding factors, as they are interrelated and can influence the rate of NICU admission. However, prior studies on singleton gestations of both small for gestational age and appropriate for gestational age fetuses have also shown that CPR is an independent predictor for NICU admissions. ${ }^{14,15}$ These studies along with our study findings of CPR- $\Delta$ at $<26$ weeks associated with NICU admission and nonreassuring fetal status requiring delivery may support the claim that CPR serves as a more sensitive marker for placental insufficiency.

Multiple second trimester ultrasound findings have been demonstrated to be associated with TTTS. ${ }^{33-35}$ Our study is the first to demonstrate an association between intertwin CPR difference and the development of TTTS. On average, intertwin CPR discordance was identified 2 weeks and 1 day prior to the development of TTTS, and 62.5\% (10/16) of pregnancies complicated by TTTS did not demonstrate an abnormal CPR value by the traditional criteria of $<1$. This temporal association of impending TTTS may prompt height- ened surveillance. Although there was a significant association of CPR- $\Delta$ at $<26$ weeks with development of TTTS, our cohort did not demonstrate significant predictive ability of the measurement. The assessment in predictive value of CPR$\Delta$ at $<26$ weeks may have been limited by the lower number of pregnancies complicated by TTTS and further studies are required to assess its clinical utility.

\section{Limitations and Strengths}

The primary limitation of this study is its retrospective design. Approximately one-third of the patients identified as MCDA twins for possible inclusion were one-time secondopinion evaluations without continued serial surveillance at our center or were evaluated prior to the inclusion of MCA evaluation into our center's surveillance protocol. As a result, the number of MCDA twins and specifically those with MCDA-specific complications that were included in the study for analysis was decreased. Additionally, delivery outcomes were unavailable for 14 patients due to delivery outside the system, but they were included in the analysis because both ultrasound and primary outcome data were available for review. Furthermore, neonatal hemoglobin and hematocrit data were not included in the analysis and antenatal diagnosis of TAPS could not be confirmed. Missing data are inherent to the retrospective nature of this study and may have resulted in a bias. Patients included in the study were identified through the search function of the clinical ultrasound database which may have led to ascertainment bias. There may also be a component of selection bias for a high-risk population, given that the patients selected for this study are from a perinatal referral center.

Overall, our study demonstrates that intertwin MCA- $\Delta_{\text {PSV- }}$ MoM is associated with TAPS, IUFD, and NICU admission, and intertwin CPR- $\Delta$ is associated with TTTS and NICU admission. We also demonstrate the strong predictive value of MCA$\Delta_{\text {PSV-MoM }}$ at $\geq 26$ weeks for TAPS with a cut-off value lower than the current diagnostic criteria for TAPS. Current recommendations from the Society for Maternal-Fetal Medicine on management of MCDA twin pregnancies do not include routine surveillance of UA or MCA Doppler during biweekly ultrasounds, but the findings from this study may suggest potential utility. ${ }^{7}$ We recognize that the overall number of complicated MCDA-twin pregnancies is lower within the study and postnatal confirmation of TAPS was not performed, which makes it difficult to make robust clinical recommendations from our study. However, the associations identified in this study brings attention to an alternative method of assessing fetal status in MCDA twin gestations through intertwin discrepancy of measurements rather than isolated values.

\section{Conclusion}

Based on these study findings, an increase in intertwin MCA and CPR discrepancy may be associated with adverse pregnancy and neonatal outcomes, including TAPS, TTTS, IUFD, and NICU admission. This study highlights the utility of 
assessing intertwin differences in Doppler indices in the surveillance of MCDA twin pregnancies and their role in potentially predicting the risk for development of perinatal complications. Earlier or more accurate identification of such complications may allow for earlier clinical interventions, including fetal therapy, evaluation for appropriate timing of delivery, allowing time for antenatal transfer to higher level of care, or ensuring delivery at a tertiary care center where adequate postnatal interventions are available. This in turn may improve neonatal outcomes. Prospective trials are required to evaluate the clinical utility of surveillance of intertwin MCA and CPR differences to identify pregnancies at risk for development of TTTS or TAPS, allowing for earlier detection and successful intervention.

\section{Note}

This work was previously presented as a poster presentation at the 28th World Congress on Ultrasound in Obstetrics and Gynecology, October 20-24, 2018, Singapore.

\section{Conflict of Interest}

L.D.P. serves on the medical advisory board of Nuvo, Jubel Health, Trice Imaging, and the Perinatal Quality Foundation. He is provided research support and is on the medical advisory board for GE Medical Systems. C.S.H. serves on the medical advisory board of Jubel Health. The remaining authors listed have no disclosures.

\section{References}

1 Hack KE, Derks JB, Elias SG, et al. Increased perinatal mortality and morbidity in monochorionic versus dichorionic twin pregnancies: clinical implications of a large Dutch cohort study. BJOG 2008;115(01):58-67

2 Gratacós E, Lewi L, Carreras E, et al. Incidence and characteristics of umbilical artery intermittent absent and/or reversed enddiastolic flow in complicated and uncomplicated monochorionic twin pregnancies. Ultrasound Obstet Gynecol 2004;23(05): $456-460$

3 Vanderheyden TM, Fichera A, Pasquini L, et al. Increased latency of absent end-diastolic flow in the umbilical artery of monochorionic twin fetuses. Ultrasound Obstet Gynecol 2005;26(01):44-49

4 Bahtiyar MO, Emery SP, Dashe JS, et al; North American Fetal Therapy Network. The North American Fetal Therapy Network consensus statement: prenatal surveillance of uncomplicated monochorionic gestations. Obstet Gynecol 2015;125(01):118-123

5 Simpson LLSociety for Maternal-Fetal Medicine. Twin-twin transfusion syndrome. Am J Obstet Gynecol 2013;208(01):3-18

6 Khalil A, Rodgers M, Baschat A, et al. ISUOG practice guidelines: role of ultrasound in twin pregnancy. Ultrasound Obstet Gynecol 2016;47(02):247-263

7 Hoskins IA, Combs CAPatient Safety and Quality Committee, Society for Maternal-Fetal Medicine. Electronic address: smfm@smfm.org. Society for Maternal-Fetal Medicine Special Statement: updated checklists for management of monochorionic twin pregnancy. Am J Obstet Gynecol 2020;223(05):B16-B20

8 Maulik D, Mundy D, Heitmann E, Maulik D. Evidence-based approach to umbilical artery Doppler fetal surveillance in high-risk pregnancies: an update. Clin Obstet Gynecol 2010;53(04):869-878

9 Maulik D, Yarlagadda AP, Youngblood JP, Willoughby L. Components of variability of umbilical arterial Doppler velocimetry-a prospective analysis. Am J Obstet Gynecol 1989;160(06): 1406-1409, discussion 1409-1412
10 Berkley E, Chauhan SP, Abuhamad ASociety for Maternal-Fetal Medicine Publications Committee. Doppler assessment of the fetus with intrauterine growth restriction. Am J Obstet Gynecol 2012;206(04):300-308

11 Unterscheider J, Daly S, Geary MP, et al. Optimizing the definition of intrauterine growth restriction: the multicenter prospective PORTO Study. Am J Obstet Gynecol 2013;208(04):290. e1-290.e6

12 Arbeille P, Roncin A, Berson M, Patat F, Pourcelot L. Exploration of the fetal cerebral blood flow by duplex Doppler-linear array system in normal and pathological pregnancies. Ultrasound Med Biol 1987;13(06):329-337

13 Flood K, Unterscheider J, Daly S, et al. The role of brain sparing in the prediction of adverse outcomes in intrauterine growth restriction: results of the multicenter PORTO Study. Am J Obstet Gynecol 2014;211(03):288.e1-288.e5

14 Khalil AA, Morales-Rosello J, Elsaddig M, et al. The association between fetal Doppler and admission to neonatal unit at term. Am J Obstet Gynecol 2015;213(01):57.e1-57.e7

15 Khalil AA, Morales-Rosello J, Morlando M, et al. Is fetal cerebroplacental ratio an independent predictor of intrapartum fetal compromise and neonatal unit admission? Am J Obstet Gynecol 2015;213(01):54.e1-54.e10

16 DeVore GR. The importance of the cerebroplacental ratio in the evaluation of fetal well-being in SGA and AGA fetuses. Am J Obstet Gynecol 2015;213(01):5-15

17 Gramellini D, Folli MC, Raboni S, Vadora E, Merialdi A. Cerebralumbilical Doppler ratio as a predictor of adverse perinatal outcome. Obstet Gynecol 1992;79(03):416-420

18 Conde-Agudelo A, Villar J, Kennedy SH, Papageorghiou AT. Predictive accuracy of cerebroplacental ratio for adverse perinatal and neurodevelopmental outcomes in suspected fetal growth restriction: systematic review and meta-analysis. Ultrasound Obstet Gynecol 2018;52(04):430-441

19 Hack KE, Kaandorp JJ, Derks JB, Elias SG, Pistorius L, Visser GH. The value of umbilical artery Doppler velocimetry in the antenatal surveillance of uncomplicated monochorionic twin pregnancies. Ultrasound Obstet Gynecol 2008;31(06):662-668

20 Pessel C, Merriam A, Vani K, et al. Do Doppler studies enhance surveillance of uncomplicated monochorionic diamniotic twins? J Ultrasound Med 2015;34(04):569-575

21 Gaziano EP, Gaziano C, Terrell CA, Hoekstra RE. The cerebroplacental Doppler ratio and neonatal outcome in diamnionic monochorionic and dichorionic twins. J Matern Fetal Med 2001;10(06): 371-375

22 Khalil AA, Khan N, Bowe S, et al. Discordance in fetal biometry and Doppler are independent predictors of the risk of perinatal loss in twin pregnancies. Am J Obstet Gynecol 2015;213(02):222.e1-222. e10

23 Stagnati V, Pagani G, Fichera A, Prefumo F. Intertwin discrepancy in middle cerebral artery peak systolic velocity and third-trimester fetal growth restriction in monochorionic-diamniotic twin pregnancy. Ultrasound Obstet Gynecol 2016;48(01):66-71

24 Dias T, Arcangeli T, Bhide A, Napolitano R, Mahsud-Dornan S, Thilaganathan B. First-trimester ultrasound determination of chorionicity in twin pregnancy. Ultrasound Obstet Gynecol 2011;38(05):530-532

25 Hadlock FP, Harrist RB, Martinez-Poyer J. In utero analysis of fetal growth: a sonographic weight standard. Radiology 1991;181(01): 129-133

26 Mari G, Deter RL, Carpenter RL, et al; Collaborative Group for Doppler Assessment of the Blood Velocity in Anemic Fetuses. Noninvasive diagnosis by Doppler ultrasonography of fetal anemia due to maternal red-cell alloimmunization. $\mathrm{N}$ Engl J Med 2000;342(01):9-14

27 Quintero RA, Morales WJ, Allen MH, Bornick PW, Johnson PK, Kruger M. Staging of twin-twin transfusion syndrome. J Perinatol 1999;19(8 Pt 1):550-555 
28 Robyr R, Lewi L, Salomon LJ, et al. Prevalence and management of late fetal complications following successful selective laser coagulation of chorionic plate anastomoses in twin-to-twin transfusion syndrome. Am J Obstet Gynecol 2006;194(03):796-803

29 Gratacós E, Lewi L, Muñoz B, et al. A classification system for selective intrauterine growth restriction in monochorionic pregnancies according to umbilical artery Doppler flow in the smaller twin. Ultrasound Obstet Gynecol 2007;30(01):28-34

30 Breathnach FM, McAuliffe FM, Geary M, et al; Perinatal Ireland Research Consortium. Definition of intertwin birth weight discordance. Obstet Gynecol 2011;118(01):94-103

31 Tollenaar LSA, Lopriore E, Middeldorp JM, et al. Improved prediction of twin anemia-polycythemia sequence by delta middle cerebral artery peak systolic velocity: new antenatal classification system. Ultrasound Obstet Gynecol 2019;53(06):788-793
32 Weingertner AS, Kohler A, Kohler M, et al. Clinical and placental characteristics in four new cases of twin anemia-polycythemia sequence. Ultrasound Obstet Gynecol 2010;35(04):490-494

33 Ong SS, Zamora J, Khan KS, Kilby MD. Prognosis for the co-twin following single-twin death: a systematic review. BJOG 2006;113 (09):992-998

34 Lewi L, Lewi P, Diemert A, et al. The role of ultrasound examination in the first trimester and at 16 weeks' gestation to predict fetal complications in monochorionic diamniotic twin pregnancies. Am J Obstet Gynecol 2008;199(05):493.e1-493.e7

35 Kusanovic JP, Romero R, Gotsch F, et al. Discordant placental echogenicity: a novel sign of impaired placental perfusion in twin-twin transfusion syndrome? J Matern Fetal Neonatal Med 2010;23(01):103-106 